\title{
Grado de acidez y potencial erosivo de las bebidas energizantes disponibles en Chile
}

\section{Acidity and erosive potential of energy drinks available in Chile}

\author{
Fresno $\mathrm{MC}^{1}$, Angel $\mathrm{P}^{1}$, Arias $\mathrm{R}^{1}$, Muñoz $\mathrm{A}^{1}$
}

\begin{abstract}
RESUMEN
Desde hace una década están disponibles en el mercado nacional, tanto bebidas como otros productos energéticos. Su popularidad ha ido en aumento, sin embargo su consumo no está exento de riesgos. La cafeína es el ingrediente activo principal de estas bebidas y el consumo excesivo puede llegar a causar alteraciones en la salud general. Mucha gente las bebe a diario sin estar consciente del daño potencial que puede significar para su salud y sus dientes, especialmente en el caso de niños y adolescentes. En Chile, la acidez de estas bebidas es desconocida. El objetivo del presente estudio fue determinar el pH de las bebidas energéticas presentes en el mercado chileno, estableciendo su potencial erosivo sobre los dientes. Metodología: La muestra quedó formada por 8 diferentes bebidas energéticas disponibles en Chile. El pH fue evaluado con un pHmetro calibrado (microprocessor pH to put AOKTON, pH/lon 510 ) a $4^{\circ} \mathrm{C}$ y $17^{\circ} \mathrm{C}$. Los resultados obtenidos fueron registrados y analizados estadísticamente. Resultados: El rango de pH osciló entre 2.57 (Kem Xtreme) y 3.30 (Red Bull). El promedio fue 2.88 a $4^{\circ} \mathrm{C}$ y 2.89 a $17^{\circ} \mathrm{C}$. Conclusiones: Todas las muestras estudiadas tuvieron $\mathrm{pH}$ ácido, haciendo de ellas bebidas potencialmente erosivas para los dientes. Los valores de $\mathrm{pH}$ fueron menores a $4^{\circ} \mathrm{C}$ que a $17^{\circ} \mathrm{C}$, pero sin diferencias estadísticamente significativas $(p>0.05)$.
\end{abstract}

Rev. Clin. Periodoncia Implantol. Rehabil. Oral Vol. 7(1); 5-7, 2014.

Palabras clave: pH, erosión dental, bebidas energéticas, potencial erosivo.

\section{ABSTRACT}

For a decade, energy drinks and other energy products have been available in the national market, and their popularity has increased. The consumption is not risk free, though. Caffeine is the main active ingredient in these drinks and an excessive consumption may be dangerous for general health. Many people drink them daily without being aware of the potential risk they pose on their health and teeth, especially on children and adolescents. Currently, the acidity of those beverages is unknown in Chile. Objectives: The aim of this study was to determine the $\mathrm{pH}$ of energy drinks, and correlate it with their erosive potential on teeth. Methodology: 8 different energy drinks available in Chile constituted the sample. $\mathrm{pH}$ was measured with a calibrated digital pHmeter (microprocessor pH to put AOKTON, pH / Ion 510) at $4^{\circ} \mathrm{C}$ and $17^{\circ} \mathrm{C}$. The results obtained were registered and statistically analyzed. Results: $\mathrm{pH}$ values ranged from 2.57 (Kem Xtreme) to 3.30 (Red Bull) the mean pH was 2.88 and 2.89 , at $4^{\circ} \mathrm{C}$ and $17^{\circ} \mathrm{C}$ respectively. Conclusions: All of the beverages showed low $\mathrm{pH}$, which makes them potentially erosive for hard dental tissue. The $\mathrm{pH}$ values were lower at $4^{\circ} \mathrm{C}$ than at $17^{\circ} \mathrm{C}$, but without statistical significance ( $p>0.05)$.

Rev. Clin. Periodoncia Implantol. Rehabil. Oral Vol. 7(1); 5-7, 2014.

Key words: pH, dental erosion, energy drinks, erosive potential.

\section{INTRODUCCIÓN}

La erosión es definida como la pérdida patológica de tejidos dentarios como resultado de la remoción causada por un agente químico cuyo $\mathrm{pH}$ sea inferior a 5.5, excluyendo pérdidas asociadas a la acción de ácidos bacterianos ${ }^{(1,2)}$. Ésta puede ser causada por ácidos intrínsecos o extrínsecos o una combinación de ellos. La erosión intrínseca es causada por ácidos gástricos e incluye reflujo gastro-esofágico y vómito recurrente como parte de cuadros de desórdenes alimentarios. Los agentes exógenos productores de erosión son variados y algunos de los más conocidos son los alimentos cítricos, bebidas carbonatadas, jugos de fruta, vinos, vinagre, derivados ácidos de leche y algunos medicamentos como vitamina C efervescente ${ }^{(3-5)}$. El potencial erosivo, es decir, la capacidad de un alimento para generar erosión dentaria ha sido estudiado en su pH, capacidad buffer, grado de saturación, concentración de calcio, concentración de fosfato e inhibidores de erosión, tales como fluoruros. Sin embargo, se ha concluido que el factor dominante en la disolución erosiva es el $\mathrm{pH}^{(6)}$

De acuerdo a la legislación chilena, bajo el reglamento sanitario de los alimentos, las bebidas energéticas son consideradas suplementos alimentarios, específicamente rotuladas como "alimentos para deportistas o bebidas energéticas o energizantes" y por ello de venta libre a disposición en el comercio. Al contener en su composición carbohidratos, guaraná, cafeína, taurina, otros aminoácidos y vitaminas, son utilizadas por jóvenes y adolescentes como alternativas naturales para mejorar el rendimiento físico y cognitivo, aumentar la concentración, atención y el estado de alerta ${ }^{(7-9)}$. Es por ello que las bebidas energéticas han experimentado un alto incremento en su consumo a nivel mundial ${ }^{(10)}$. Chile no está ajeno a esta tendencia, de acuerdo al último reporte entregado por la Asociación Nacional de Bebidas Refrescantes (ANBER), durante los últimos diez años la evolución de esta categoría ha tenido un crecimiento exponencial. De hecho, considerando desde el año 2006 hasta la fecha, la venta de bebidas energéticas ha crecido en un promedio de $80 \%$; hoy se consumen alrededor de 0.6 litros de estas bebidas per cápita al año, aunque lejos aún de la realidad que muestran países como Estados Unidos, donde el consumo por persona alcanza los cuatro litros anuales.

El objetivo de este estudio descriptivo experimental fue determinar el $\mathrm{pH}$ de las bebidas energizantes y correlacionarlo con el potencial erosivo sobre los dientes.

1. DDS. Área Operatoria Dental, Departamento de Odontología Restauradora. Facultad de Odontología, Universidad de Chile. Chile. 


\section{MATERIAL Y MÉTODO}

Se seleccionaron 8 diferentes bebidas energéticas disponibles comercialmente la ciudad de Santiago de Chile, adquiriéndose 3 latas de cada una de ellas seleccionadas al azar y provenientes de diferentes lotes de producción, lo que se corroboró con la numeración y fecha indicada en el envase, quedando constituida la muestra por 24 latas.

Se formaron 8 grupos experimentales de 3 bebidas cada uno, separados arbitrariamente por marca comercial: Grupo A: Dark Dog Free; Grupo B: Kem Xtreme; Grupo C: Speed Unlimited; Grupo D: Red Bull; Grupo E: Adrenaline, Sobe Rush; Grupo F: Speed Injection, Reloaded; Grupo G: Battery y Grupo H: Dark Dog.

Los datos fueron obtenidos y registrados por un solo examinador calibrado. Para la medición de $\mathrm{pH}$ se obtuvieron 12 muestras de $40 \mathrm{ml}$ para cada grupo, con un total de 96 especímenes de prueba. Seis de ellas se midieron con la bebida a $17^{\circ} \mathrm{C}$ y las otras seis a $4^{\circ} \mathrm{C}$ en cada tipo de bebida, ya que son las temperaturas habituales de ingesta, para lo cual las muestras fueron mantenidas almacenadas a las temperaturas mencionadas. El pH fue medido usando un pHmetro digital calibrado microprocessor pH meter AOKTON, pH/lon 510 (Cole-Parmer 625 East Bunker Court Vernon Hills, IL 60061 USA).

Para cada grupo se obtuvieron los promedios y desviaciones estandar de $\mathrm{pH}$ según temperatura y los datos fueron vaciados en una planilla Excel y analizados estadísticamente con test t Student utilizando el programa IBM, SPSS Statistics, versión 19.

\section{RESULTADOS}

Todas las bebidas de la muestra presentaron $\mathrm{pH}<5.5$ considerado como crítico con un rango entre $2.57-3.26$ a $4^{\circ} \mathrm{C}$ y 2.60 3.30 a $17^{\circ} \mathrm{C}$. El menor fue para Kem Xtreme a $4^{\circ} \mathrm{C}(\mathrm{pH}=2.57)$ y el mayor Red Bull a $17^{\circ} \mathrm{C}(\mathrm{pH}=3.30)$

Los resultados de $\mathrm{pH}$ de las bebidas energéticas a diferentes temperaturas estudiadas se resumen en la Tabla 1. El pH promedio fue 2.88 a $4^{\circ} \mathrm{C}$ y 2.89 a $17^{\circ} \mathrm{C}$, sin diferencias estadísticamente significativas $(p>0.05)$.

Tabla 1. Promedio pH para cada grupo de bebidas energizantes.

\begin{tabular}{|l|c|c|}
\hline Bebida & $\mathbf{4}^{\circ} \mathrm{C}$ & $17^{\circ} \mathrm{C}$ \\
\hline Dark Dog Free & 3.11 & 3.15 \\
\hline Kem Xtreme & 2.57 & 2.60 \\
\hline Speed Unlimited & 2.77 & 2.77 \\
\hline Red Bull & 3.26 & 3.30 \\
\hline Adrenaline & 3.11 & 3.11 \\
\hline Speed Inyection Reloaded & 2.60 & 2.67 \\
\hline Battery & 2.72 & 2.73 \\
\hline Dark Dog & 2.88 & 2.81 \\
\hline Promedio & 2.88 & 2.89 \\
\hline Desviación estándar & 0.259 & 0.257 \\
\hline T test & $\mathrm{p}=0.343$ & \\
\hline
\end{tabular}

\section{DISCUSIÓN}

Los datos epidemiológicos sugieren que la erosión dental es un importante problema de salud pública en el mundo(1,11-13). En Chile no hay datos sobre ello pero si se sabe que el aumento en el consumo de bebidas de fantasía ha aumentado en forma exponencial en los últimos años. Entre ellas se encuentran las bebidas energizantes ${ }^{(14)}$, que fueron introducidas en 1987 en Austria y a fines de los 90 en EE.UU.
En Chile son comercializadas hace más de una década y debido a su composición, el Reglamento Sanitario vigente las rotula como "alimentos para deportistas" por lo tanto son de libre disposición y venta, orientadas a un público básicamente joven. Éstas han sido utilizadas por ellos para incrementar la resistencia física, mejorar la concentración, prolongar las horas de vigilia promoviendo el estado de alerta, proveer sensación de bienestar y estimular el metabolismo por su contenido de cafeína, taurina, guaraná y otras sustancias fisiológicamente activas.

Debido a lo anterior, su consumo es muy popular entre estudiantes universitarios y deportistas ${ }^{(7,8,15-18)}$. Estas bebidas son utilizadas a diario entre los adolescentes y adultos jóvenes, sin embargo no se han planteado cuales son los efectos negativos que sus componentes pudieran tener sobre la salud general y dental, en particular.

El ejercicio está siendo cada vez más popular debido a sus beneficios para el bienestar físico, también es promovido para la prevención de la obesidad. La ingesta de líquidos durante y después de éste repone la pérdida de fluidos, minimizando los efectos dañinos de la deshidratación en la dinámica cardiovascular, la temperatura corporal y el rendimiento deportivo, en el momento en que las personas realizan actividad deportiva, está disminuida en ellas la capacidad protectora salival, ya que al producirse deshidratación como parte de su entrenamiento, la pérdida de fluidos corporales aumenta por aumento de temperatura, sudoración y respiración bucal. Esto determina que la secreción salival disminuya en forma significativa, aumentando el riesgo erosivo(10,19)

La erosión dental está relacionado con las fuentes de ácidos intrínsecos y extrínsecos, con las características estructurales de los dientes y también con las propiedades fisiológicas de la saliva. Los alimentos y bebidas acídicas pueden afectar los dientes y su exposición crónica a menudo conduce al desarrollo de un debilitamiento de la estructura dentaria (atrición, erosión y caries) ${ }^{(20)}$. Estudios clínicos muestran que la exposición a los ácidos, combinados con un flujo salival disminuido, dan como resultado una mayor disolución de los tejidos dentarios ${ }^{(21)}$. En periodos de mayor estres, como sucede a los estudiantes en periodo de exámenes, el flujo salival está disminuido (por acción del sistema nervioso simpático), por lo tanto su factor protector es menor lo que favorece la acción erosiva de los ácidos sobre la superficie dentaria.

Cabe destacar que dentro de la composición de las bebidas energéticas están los ácidos cítrico (ácido orgánico) y fosfórico (ácido inorgánico), los cuales provocan lesiones erosivas en los tejidos dentarios; estos ácidos también se encuentran en las bebidas colas ${ }^{(22,23)}$. Por otro lado, la expresión del contenido ácido $(\mathrm{pH})$, en conjunto con la capacidad buffer y el contenido de iones ácidos disponibles, son los mayores responsables de la producción de erosiones, ya que sin un ambiente ácido los otros componentes no son $\operatorname{activos}^{(23)}$.

Aunque el potencial erosivo de las bebidas depende de una interrelación compleja de numerosos factores tales como: El tipo de ácido, su concentración, temperatura, tiempo de permanencia de la bebida en la boca, capacidad buffer de la saliva. Hoy en día se acepta como indicadores válidos tanto la titulación ácida como la medición de $\mathrm{pH}$, siendo este último el más utilizado ${ }^{(6,24-26)}$. Los resultados del presente estudio demuestran que el $\mathrm{pH}$ de las bebidas energéticas en nuestro medio tendrían un alto potencial erosivo debido a su acidez expresada en el bajo $\mathrm{pH}$ promedio, corroborando lo encontrado por Cavalcanti(27). Hoy en día se puede afirmar que la acidez es uno de los factores etiológicos mas importantes en la erosión dental en adolescentes, esto sustentado en numerosos estudios en los cuales se establece una relación directa entre la acidez de la bebida y la pérdida de tejido dentario(10,28-32). Este hecho generalmente es desconocido por los consumidores, ya que no existe obligación legal en nuestro país de especificar los valores de acidez en el envase.

Dada la correlación positiva entre erosión, reblandecimiento de la superficie dentaria y temperatura, se sugiere el consumo de bebidas a la temperatura más baja posible ${ }^{(33)}$. Sin embargo, bajo las condiciones del presente estudio, en que las temperaturas evaluadas corresponden a las que habitualmente se ingieren, la temperatura no modificó significativamente el $\mathrm{pH}$.

Algunos estudios demuestran que las bebidas pueden ser modificadas con adición de calcio y fosfato como una medida que ayuda a reducir el potencial erosivo de ellas, permitiendo que su ingesta sea más segura ${ }^{(26,28,34)}$. Sin embargo, una forma sencilla de prevenir o minimizar sus efectos sobre las superficies de los dientes, es conocer su potencial erosivo para educar y prevenir los efectos deletéreos de una ingesta excesiva de ellas ${ }^{(26)}$. 
Los resultados obtenidos sugieren que todas las bebidas evaluadas, son potencialmente erosivas sobre los tejidos dentarios debido a su acidez, pero se debe tener en consideración que en los estudios realizados en laboratorio se tiene un mayor control de las posibles variables, esto podría interferir con los resultados obtenidos, por lo tanto, vale la pena señalar que entre las limitaciones de este tipo de estudio está la incapacidad de extrapolar completamente las conclusiones a los seres humanos ${ }^{(27)}$. Factores inherentes a la saliva tales como composición, potencial remineralizante, capacidad amortiguadora y flujo podrían actuar como factores protectores sobre los tejidos dentarios. Es necesario destacar la importancia y necesidad de estudios adicionales in situ, para que pueda conocerse el verdadero efecto nocivo de estas bebidas para los dientes.

\section{CONCLUSIONES}

Todas las bebidas estudiadas presentaron $\mathrm{pH}$ ácido, haciendo de ellas bebidas potencialmente erosivas para los dientes. Los valores de $\mathrm{pH}$ fueron menores a $4^{\circ} \mathrm{C}$ que los medidos a $17^{\circ} \mathrm{C}$, pero sin diferencias estadísticamente significativas ( $p>0.343$ ).
Los valores de acidez no aparecen especificados en los envases y se sugiere considerarlos como información importante para la población que consume este tipo de bebidas.

Al ser la erosión un problema de salud pública, es nuestro rol como profesionales odontólogos hacer notar estas falencias en los componentes y etiquetado, sugiriendo a la industria las modificaciones necesarias para educar a la población y desarrollar productos más saludables.

\section{CONFLICTO DE INTERESES} ningún tipo.

Los autores declaran no presentar conflictos de interés de

\section{REFERENCIAS BIBLIOGRÁFICAS}

1. Lussi A, Jaeggi T, Zero D. The role of diet in the aetiology of dental erosion. Caries Res, 2004; 38 Suppl 1: 34-44.

2. Smith BG, Bartlett DW, Robb ND. The prevalence, etiology and management of tooth wear in the United Kingdom. J Prosthet Dent, 1997; 78(4): 367-372.

3. Jarvinen VK, Rytomaa II, Heinonen OP. Risk factors in dental erosion. J Dent Res, 1991; 70(6): 942-947.

4. ten Cate JM, Imfeld T. Dental erosion, summary. Eur J Oral Sci, 1996; 104(2 Pt 2): $241-244$

5. Jaeggi T, Lussi A. Prevalence, incidence and distribution of erosion. Monogr Oral Sci, 2006; 20: 44-65

6. Barbour ME, Lussi A, Shellis RP. Screening and prediction of erosive potential. Caries Res, 2011; 45 Suppl 1: 24-32.

7. Duchan E, Patel ND, Feucht C. Energy drinks: A review of use and safety for athletes. Phys Sportsmed, 2010; 38(2): 171-179.

8. Kaminer Y. Problematic use of energy drinks by adolescents. Child Adolesc Psychiatr Clin N Am, 2010; 19(3): 643-650.

9. Seidl $\mathrm{R}$ et al. A taurine and caffeine-containing drink stimulates cognitive performance and well-being. Amino Acids, 2000; 19(3-4): 635-642.

10. Coombes JS. Sports drinks and dental erosion. Am J Dent, 2005; 18(2): 101-104. 11. Dugmore CR, Rock WP. The prevalence of tooth erosion in 12-year-old children. Br Dent J, 2004; 196(5): 279-282; discussion 273.

12. Al-Majed I, Maguire A, Murray JJ. Risk factors for dental erosion in 5-6 year old and 12-14 year old boys in Saudi Arabia. Community Dent Oral Epidemiol, 2002; 30(1): $38-46$

13. Boulze D, Montastruc $P$, Cabanac M. Water intake, pleasure and water temperature in humans. Physiol Behav, 1983; 30(1): 97-102.

14. Biblioteca del Congreso Nacional de Chile: Crece consumo de bebidas deportivas. 2011; Available from: www.bcn.cl/come_inteligente/come_inteligente/ crece consumo bebidas deportivas.

15. Malinauskas BM et al. A survey of energy drink consumption patterns among college students. Nutr J, 2007; $6: 35$.

16. Arria $\mathrm{AM}$ et al. Energy drink consumption and increased risk for alcohol dependence. Alcohol Clin Exp Res, 2011; 35(2): 365-375

17. Seifert SM et al. Health effects of energy drinks on children, adolescents, and young adults. Pediatrics, 2011; 127(3): 511-528.

18. Attila S, Cakir B. Energy-drink consumption in college students and associated factors. Nutrition, 2011; 27(3): 316-322.
19. Coombes JS, Hamilton KL. The effectiveness of commercially available sports drinks. Sports Med, 2000; 29(3): 181-209.

20. Touyz LZG MA. Dental ravages from acidulated soft drinks. Journal of Aesthetic and Implant Dentistry, 2006; 8(3): 20-33.

21. Lussi $A$ et al. Dental erosion-an overview with emphasis on chemical and histopathological aspects. Caries Res, 2011; 45 Suppl 1: 2-12.

22. Imfeld T. Evaluation of the cariogenicity of confectionery by intra-oral wiretelemetry. SSO Schweiz Monatsschr Zahnheilkd, 1977; 87(5): 437-464.

23. Borjian A et al. Pop-cola acids and tooth erosion: An in vitro, in vivo, electronmicroscopic, and clinical report. Int J Dent, 2010 (2010); Article ID 957842, 12 pages.

24. Benjakul PCC. Association of dental enamel loss with the $\mathrm{pH}$ and titratable acidity of beverages. J Dent Sci, 2011; 6: 129-133.

25. Murrell $S$ et al. Comparison of in vitro erosion potentials between beverages available in the United Kingdom and the United States. J Dent, 2010; 38(4): 284-289. 26. Manton DJ et al. Effect of casein phosphopeptide-amorphous calcium phosphate added to acidic beverages on enamel erosion in vitro. Aust Dent J, 2010; 55(3): 275-279. 27. Cavalcanti AL et al. Short communication: In vitro assessment of erosive potential of energy drinks. Eur Arch Paediatr Dent, 2010; 11(5): 253-255.

28. Larsen MJ, Nyvad B. Enamel erosion by some soft drinks and orange juices relative to their $\mathrm{pH}$, buffering effect and contents of calcium phosphate. Caries Res, 1999; 33(1): 81-87.

29. Rees J, Loyn T, McAndrew R. The acidic and erosive potential of five sports drinks. Eur J Prosthodont Restor Dent, 2005; 13(4): 186-190.

30. Ehlen LA et al. Acidic beverages increase the risk of in vitro tooth erosion. Nutr Res, 2008; 28(5): 299-303.

31. Seow WK, Thong KM. Erosive effects of common beverages on extracted premolar teeth. Aust Dent J, 2005; 50(3): 173-178; quiz 211.

32. Owens BM. The potential effects of $\mathrm{pH}$ and buffering capacity on dental erosion. Gen Dent, 2007; 55(6): 527-531.

33. Barbour ME et al. The relationship between enamel softening and erosion caused by soft drinks at a range of temperatures. J Dent, 2006; 34(3): 207-213.

34. Jensdottir T, Bardow A, Holbrook P. Properties and modification of soft drinks in relation to their erosive potential in vitro. J Dent, 2005; 33(7): 569-575.

35. Cavalcanti AL et al. Avaliação in vitro do potencial erosivo de bebidas isotônicas. Rev Bras Med Esporte, 2010; 16: 455-458. 